\title{
Social cognition and Theory of Mind: controversies and promises for understanding major psychiatric disorders
}

\author{
Flavio Eduardo Martins-Junior ${ }^{1,2}$, Breno Sanvicente-Vieira ${ }^{3}$, Rodrigo Grassi-Oliveira ${ }^{3}$ and Elisa \\ Brietzke $^{1}$ \\ 1- Universidade Federal de São Paulo, São Paulo, SP, Brazil \\ 2- Eli Lilly and Company, Global Patient Safety, São Paulo, SP, Brazil \\ 3- Pontifícia Universidade Católica do Rio Grande do Sul, Porto Alegre, RS, Brazil
}

\begin{abstract}
The term "social cognition" generally refers to the mental operations that underlie social interactions including the perception and interpretation of the intentions, dispositions, and behaviors of others and the generation of a response to these behaviors. Social cognition has been considered a valuable and promising field that strives to understand the nature and outcome of major mental disorders. This article discusses the concept of social cognition and its relationship to Theory of Mind (ToM). Theory of Mind in autistic spectrum disorders has been studied since the 1980s, and cognitive impairments in these disorders may be restricted to ToM deficits because other cognitive domains and nonsocial intelligence are preserved. This article addresses the issues of human ontogeny, reviews the main findings from research on schizophrenia and bipolar disorder, and summarizes the tools commonly used in the assessment of these illnesses. Keywords: social cognition, Theory of Mind, bipolar disorder, schizophrenia.
\end{abstract}

Received 4 May 2011; received in revised form 19 October 2011; accepted 12 November 2011. Available online 29 December 2011

\section{Introduction}

The term "social cognition" has various meanings, but it generally refers to the mental operations that underlie social interactions including the perception and interpretation of the intentions, dispositions, and behaviors of others, and the generation of a response to these behaviors (Green et al., 2008). Social cognitive processes in humans describe the ways individuals draw inferences about other people's beliefs and the ways they weigh social situational factors in making these inferences (Green et al., 2008). Although social cognition is a valuable and promising field that attempts to understand the nature and outcomes of major mental disorders, lack of agreement on the myriad of terms, definitions, and

Flavio Eduardo Martins-Junior and Elisa Brietzke, Program for Recognition and Intervention in Individuals at Risk Mental States, Department of Psychiatry, Universidade Federal de São Paulo, São Paulo, Brazil. Breno Sanvicente-Vieira and Rodrigo Grassi-Oliveira, Group of Developmental Cognitive Neuroscience, Faculty of Psychology, Pontifícia Universidade Católica do Rio Grande do Sul, Porto Alegre, Brazil. Flavio Eduardo Martins Junior, Eli Lilly and Company, Global Patient Safety, São Paulo, Brazil. Correspondence regarding this article should be directed to: Flavio Eduardo Martins Junior, Rua Machado Bittencourt, 222, Vila Clementino, CEP 044000-400, São Paulo SP, Brazil. E-mail: femj_@hotmail.com measurements associated with this field creates problems for theorists. Moreover, as Macrae (2001) indicated in his work, social cognition is one of the most studied themes in current experimental psychology research.

Among the confusion in the literature about the various terms associated with social cognitive theory, the Theory of Mind (ToM) is one of the most discussed. It refers to the ability to infer mental states (e.g., beliefs, desires, intentions, imagination, emotions) that cause actions. An individual with a ToM is able to reflect on the content of his/her own and others' minds (Baron-Cohen, 2001).

Because social cognition and ToM belong to a special cognitive domain that directly reflects the interactions within a social environment, thus affecting quality of life, we can hypothesize that these functions are compromised in the case of mental illnesses characterized by cognitive impairments or disabilities (e.g., schizophrenia and bipolar disorder) (Couture, 2011). The study of cognition in major psychiatric disorders is an important research topic, but there is a paucity of available literature that meticulously addresses these issues.

\section{Methods and objectives}

We present a narrative review that focuses on social cognition and its relationship to ToM, clarifying the ambiguity in the concepts in the literature. Another 
objective is to discuss the tendencies in the assessment of ToM and to review the populations (i.e., ages, species, psychopathologies) with impairments in this ability.

\section{Social cognition and Theory of Mind}

Three broad fields of knowledge appear to cover everything humans can know or conceive of knowing. The first is knowledge of the nonsocial environment, consisting of inanimate objects that do not interact. The second and third areas are the knowledge of others' minds and our own. Humans share neurobiological and psychological mechanisms with other mammalian species; however, the abilities that arise from the knowledge of our own mind are much more refined (Adolphs, 2009).

Social cognition refers to the cognitive processes related to the perception and interpretation of the stimuli around us to develop the knowledge we have about our own mind and others' minds. One way to address the major themes and findings across areas of social cognitive research is to divide these domains into the understanding of others (e.g., ToM, empathy), selfunderstanding (e.g., visual self-recognition, agency), self-control (e.g., impulse control, reappraisal), and the interface between self and others. The current theory of these processes proposes a dichotomous scheme. On one end of the spectrum are responses that are exclusively controlled, conscious, and deliberate (e.g., self-reflection). On the other end of the spectrum are the responses that are automatic, unintentional, and unconsidered (e.g., feeling rejected). Between these extremes are processes that engage or mediate controlled and automatic responses (e.g., reappraisal, ToM) (Lieberman, 2007). Emotional regulation, for example, often involves the initiation of new emotional responses or the alteration of ongoing responses through volitional regulatory actions that are used to influence the regulatory process. Failures to adaptively regulate emotional responses can be related to some psychiatric disorders (Ochsner \& Gross, 2005).

The term "Theory of Mind" was originally proposed in a seminal article from the late 1970s by primatologists Premack and Woodruff (1978) who suggested that chimpanzees may be capable of inferring the mental states of others from the same species. During the last two decades of the 20th century, the concept was adopted to explain behavioral symptoms in autistic spectrum disorders (Baron-Cohen, Leslie, \& Frith, 1985) and schizophrenia (Frith, 1992). Today it is known as the ability to reason the mental states and emotions of other persons (Fernyhough, 2008).

The ontogeny of ToM faculties does not differ greatly from the maturation of other brain functions. The ability to appreciate one's own and others' mental states follows a distinct sequence of acquisition (Brüne \& Brüne-Cohrs, 2006). In the early months of life, infants primarily engage in dyadic, face-to-face interactions.
By the second month, they begin to focus on the eyes and mouths of other people and become distressed when others pose a "still face" and cease interacting with them. At the end of the first year, infants develop joint attention and are able to engage in triadic personobject-person interactions (Striano \& Reid, 2006). The typical 24-month-old child can engage in "pretend play" using skills to understand that the other person knows he/she is just pretending. By 3 years of age, a normal child can understand that merely touching a box is not enough to know what is inside. At the age of 4 years, typical children pass the "false belief" test, recognizing when someone has a false belief about the world. Not until 9 years of age does the typical infant recognize faux pas, what may hurt others' feelings and what may, therefore, be better left unspoken (Baron-Cohen, 2008). Evidence suggests that the social brain continues its functional development during adolescence, a period characterized by social change, heightened selfconsciousness, increased importance and complexity of peer relationships, and improved understanding of others (Blakemore, 2008).

The modular perspective of the ontogeny of ToM hypothesizes that its development depends on the neurological maturation of the involved brain regions. Experience may activate ToM mechanisms, but it does not determine its structure. This perspective implies the static and inflexible development of this cognitive ability (Brüne \& Brüne-Cohrs, 2006). Data support the theory that the extent and nature of the social interaction that children experience may influence the development of their social understanding (Carpendale $\&$ Lewis, 2004). Two other hypothetical models (i.e., metarepresentational theory and simulation theory) propose a greater role for experience in the development of individual ToM abilities, but these theories place less importance on intrinsic nervous system maturation (Brüne \& Brüne-Cohrs, 2006).

Still unknown is whether humans have truly unique cognitive properties. Experts recognize aspects of imitation, ToM, language, and consciousness in other primates. This possibility would mean that these human abilities result not from qualitative differences but from the combination and improvement of these abilities. Functional brain imaging studies in humans have identified cortical areas related to empathy, ToM, imitation, and the distinction between self and others (Roth \& Dicke, 2005). Although this evidence suggests the existence of brain structures specialized in processing social information in contrast to the idea that ToM would emerge from processes of other cognitive domains, evidence on this matter remains inconclusive.

\section{Social cognition and major psychiatric disorders}

Theory of mind in autistic spectrum disorders has been studied since the 1980s, and cognitive impairments in 
these disorders may be restricted to ToM deficits because other cognitive domains and nonsocial intelligence are preserved (Baron-Cohen, 2001). Classic autism and Asperger's syndrome share three core diagnostic features: (i) difficulties in social and communicative development, (ii) unusually strong, narrow interests, and (iii) repetitive behavior (Baron-Cohen, 2008).

In 1992, Frith proposed that the formal thought disturbances observed in schizophrenia, a core psychopathological cluster of this disorder, may be related to ToM deficits (Frith, 1992). Because bipolar disorder and schizophrenia share some psychopathological clusters, a reasonable hypothesis is that the same assumption may be valid in both cases. This inference is strengthened by the fact that ToM-related issues addressed in studies that involve these disorders are almost identical. Today, psychopathological clusters are addressed as domains and not as an unambiguous feature of a specific mental disorder (Maia \& Frank, 2011). Consequently, cognitive impairments in some major mental illnesses may be the result of common abnormal brain function rather than dysfunction of brain regions specific to one disorder (Maia \& Frank, 2011).

The first ToM studies of schizophrenia were reported during the early 1990s. Despite the large amount of evidence accumulated during this period that suggested ToM impairments in this disorder, it is unclear whether these impairments may occur separately from global cognitive decline. Also uncertain is how they might fluctuate with acuity or chronicity (Brüne, 2005). First-degree relatives of patients with schizophrenia usually perform poorly in social cognition tasks compared with healthy controls (Janssen, Krabbendam, Jolles, \& van Os, 2003; Mazza, Di Michele, Pollice, Casacchia, \& Roncone, 2008). An assessment that involved adolescents who were genetically at high risk for schizophrenia showed social skill impairments but no ToM deficits (Gibson, Penn, Prinstein, Perkins, \& Belger, 2010). The extent of the association between ToM abilities and realworld functional outcomes in schizophrenia remains inconclusive (Couture, Granholm, \& Fish, 2011; Sparks, McDonald, Lino, O’Donnell, \& Green, 2010).

Social cognition status among subjects with bipolar disorder is even less clear, and available data on this matter are scarce. Also unclear is whether the ToM deficits observed in studies of patients who experience manic or depressive episodes remain after acute phase remission (Kerr, Dunbar, \& Bentall, 2003; Olley, Malhi, Bachelor, Cahill, Mitchell, \& Berk, 2005). Nonsocial cognition may also be a confounding factor in bipolar disorder. One study reported no significant difference in the performance on ToM tasks between euthymic patients and healthy controls after correction analysis for executive function deficits (Bora et al., 2005).

\section{Assessment of Theory of Mind}

Theory of Mind assessment continues to develop (Bora, Yucel, \& Pantelis, 2009; Sprong, Schothorst, Vos, Hox, \& van Engeland, 2007). The tests and tasks already developed often focus on clinical populations in which the performance would be inferior to the general population (Bora et al., 2009; Bosco, Colle, De Fazio, Bono, Ruberti, \& Tirassa, 2009; Brüne \& Brüne-Cohrs, 2006). The main challenge of ToM assessment is the conceptual divergence in the understanding of this function (Meltzoff, 1999; Sprong et al., 2007) reflected by the various tools used to evaluate ToM. Because of this divergence, the literature includes studies that utilized a variety of tests, tasks, and assessment methods for ToM (Brüne \& Brüne-Cohrs, 2006).

The simplest test that was developed in the first stage of ToM research was the false-belief paradigm, which measures an individual's ability to recognize the false beliefs of others (Brüne \& Brüne-Cohrs, 2006; Müller \& Liebermann, 2004; Sprong et al., 2007). In one example, a famous singer is on tour and is coming to your city for a performance scheduled for next Sunday. On Wednesday, however, you hear on the radio that the show has been rescheduled for next week. Your friend suggests that you should go together to the show this week. You recognize that your friend has a false belief (i.e., he/she thinks the performance is still scheduled on Sunday), and you know your friend thinks this because he/she did not hear the news that the show has been rescheduled.

The two most common tests that use this paradigm are the Sally-and-Anne Test and the Smarties Test. The Sally-and-Anne Test is a classic task developed by Wimmer \& Perner (1983) in which a situation is presented to a participant who has to predict the behavior of a character using information contained in a short story. This simple test is useful for assessing the capacity of "metarepresentation." Children under 4 years of age usually have poor results, most likely because they cannot understand false beliefs (Brüne \& Brüne-Cohrs, 2006). The Smarties Test assesses the ability of a child to notice an individual's false beliefs. Perner, Frith, Leslie, \& Leekman (1989) developed a scenario in which the participant is offered three boxes of a specific item such as cookies. However, the boxes actually contain a different item such as scissors. The participant is then asked what an individual who they do not know would think was inside the box. Other tasks, in addition to the Smarties Test and Sally-and-Anne Test, utilize the false-belief paradigm. Examples include Happé's Strange Stories (Happé, 1994), false-belief picture sequencing (Brüne \& Bodenstein, 2005), Subjective ToM Assessment (Duval, Piolino, Bejanin, Eustache, \& Desgranges, 2010), the Maxi Test (Wimmer \& Perner, 1983), False-Belief Stories (Frith \& Corcoran, 1996), and the ToM Test (Muris et al., 1999). 
After the development of these instruments, more sophisticated paradigms such as metaphor, irony, and faux pas were developed (Brüne \& Brüne-Cohrs, 2006; Frith \& Corcoran, 1996; Muris et al., 1999). These paradigms also involve a variety of tests and tasks, which usually use stories that involve double-bluff, mistakes, persuasion, or white lies (Happé, 1994). Presenting the stimuli with cartoons is common. In one meta-analysis performed by Bora et al. (2009), the Hinting Test had greater statistical results than the other tests that used stories with higher-order false beliefs. In the Hinting Test, the participant has to understand the meaning of a story, but the stimuli are presented in indirect speech (Frith \& Corcoran, 1996). Happé (1994) developed the Theory of Mind Advanced Test, which is composed of stories and drawings. Following the presentation of a story, the participant is asked to answer a series of openended questions. The test is a useful measure of healthy subjects compared with autistic patients.

Because the cognitive understanding of ToM also extends to other abilities (Sabbagh, Moulson, \& Harkness, 2004), theorists developed tests that measure the capacity to read others to understand an emotional state by reading the eyes or facial cues (Baron-Cohen, Wheelwright, Hill, Raste, \& Plumb, 2001). This paradigm involves more automatic skills, and it has been called socioperceptual or mental state decoding (Bora, Eryavuz, Kayahan, Sungu, \& Veznedaroglu, 2006; Gokcen, Bora, Erermis, Kesikci, \& Aydin, 2009; Sabbagh et al., 2004). In the Face Test (Adolphs, 2002), the participant is asked to look at a picture of a face and name which of seven basic emotions it represents (i.e., happiness, fear, surprise, disgust, sadness, anger, and distress). Composed of 36 pictures, the Eyes Test is used to test for the decoding of mental states. The adaptation of Baron-Cohen (2001) has 27 pictures and has been utilized in several scientific studies (Gokcen et al., 2009). The test asks the subject to name the mental state of the person in the picture, and the picture shows just the eye region.

In addition to these paradigms and tests, examiners also use subjective assessment, which is generally performed using scales. Development of the scales is done with aspects of or questions from other interviews (Duval et al., 2010). The best example is the Theory of Mind Assessment Scale (Bosco et al., 2009; Wellman, \& Liu, 2004).

\section{Conclusion}

Many unresolved questions remain regarding the relationship between major psychiatric disorders such as schizophrenia and bipolar disorder, and social cognition, independent from global executive function. However, some points have become clear with recent studies. Measures of ToM may not always reflect the same ability when the assessment tools (e.g., the Eye Test and faux pas) are different, and it may not necessarily engage the same faculties and brain networks. Many of these tasks have odd paradigms that utilize false-belief tasks and assess emotion recognition. A report from the U.S. National Institute of Mental Health workshop on social cognition in schizophrenia established that future key research topics should address the factor structure within social cognition, the relationship between it and basic cognition, and alternative assessment approaches. This report pointed to the future tendency of identifying the timing of social cognitive impairment in relation to the disorder's longitudinal course and connection between social cognition and neural circuits (Green et al., 2008). Studies indicated severe ToM deficits in autistic and schizophrenic patients beyond the tendency of other mental disorders to likely have impairments in ToM or social cognition. An article from the International Society for Bipolar Disorder proposed a battery for cognitive assessment. The authors concluded that social cognition is increasingly implicated in bipolar disorder and suggested that new studies should evaluate the tests that assess this disorder (Yatham et al., 2010). Even with scarce empirical studies, social cognition should follow the same trajectory of neurodevelopment.

The findings suggest that an effective mental treatment cannot be obtained without addressing collective behavior. Humans are essentially a social species. The clarification of these issues is a crucial step toward developing an intervention that is capable of mitigating social cognition impairments.

\section{References}

Adolphs, R. (2002). Recognizing emotion from facial expressions: Psychological and neurological mechanisms. Behavioral and Cognitive Neuroscience Reviews, 1(1), 21-62.

Adolphs, R. (2009). The social brain: Neural basis of social knowledge. Annual Review of Psychology, 60, 693-716.

Baron-Cohen, S, Leslie, A. M., \& Frith, U. (1985). Does the autistic child have a "theory of mind"? Cognition, 21(1), 37-46.

Baron-Cohen, S, Wheelwright, S., Hill, J., Raste, Y., \& Plumb, I. (2001). The "Reading the Mind in the Eyes" Test revised version: A study with normal adults, and adults with Asperger syndrome or high-functioning autism. Journal of Child Psychology and Psychiatry, 42(2), 241-251.

Baron-Cohen, S. (2001). Theory of mind in normal development and autism. Prisme, 34, 174-183.

Baron-Cohen, S. (2008). Theories of the autistic mind. Psychologist, 21(2), 112-116.

Blakemore, S. J. (2008). The social brain in adolescence. Nature Reviews Neuroscience, 9(4), 267-277.

Bora, E, Vahip, S., Gonul, A. S., Akdeniz, F., Alkan, M., Ogut, M., \& Eryavuz, A. (2005). Evidence for theory of mind deficits in euthymic patients with bipolar disorder. Acta Psychiatrica Scandinavica, 112(2), 110-116.

Bora, E., Eryavuz, A., Kayahan, B., Sungu, G., \& Veznedaroglu, B. (2006). Social functioning, theory of mind and neurocognition in outpatients with schizophrenia; mental state decoding may be a better predictor of social functioning than mental state reasoning. Psychiatry Research, 145(2-3), 95-103.

Bora, E., Yucel, M., \& Pantelis, C. (2009). Theory of mind impairment in schizophrenia: Meta-analysis. Schizophrenia Research, 109(1-3), 1-9.

Bosco, F. M., Colle, L., De Fazio, S., Bono, A., Ruberti, S., \& Tirassa, M. (2009). Th.o.m.a.s.: An exploratory assessment of Theory of 
Mind in schizophrenic subjects. Consciousness and Cognition, 18(1), 306-319.

Brüne, M. (2005). "Theory of mind" in schizophrenia: A review of the literature. Schizophrenia Bulletin, 31(1), 21-42.

Brüne, M., \& Bodenstein, L. (2005). Proverb comprehension reconsidered: "theory of mind" and the pragmatic use of language in schizophrenia. Schizophrenia Research, 75(2-3), 233-239.

Brüne, M., \& Brüne-Cohrs, U. (2006). Theory of mind: evolution, ontogeny, brain mechanisms and psychopathology. Neuroscience and Biobehavioral Reviews, 30(4), 437-455.

Carpendale, J. I. M., \& Lewis, C. (2004). Constructing an understanding of mind: The development of children's social understanding within social interaction. Behavioral and Brain Sciences, 27(1), 79-96; discussion 96-151.

Couture, S. M., Granholm, E. L., \& Fish, S. C. (2011). A path model investigation of neurocognition, theory of mind, social competence, negative symptoms and real-world functioning in schizophrenia. Schizophrenia Research, 125(2-3), 152-160.

Duval, C., Piolino, P., Bejanin, A., Eustache, F., \& Desgranges, B. (2010). Age effects on different components of theory of mind. Consciousness and Cognition, 20(3), 627-642.

Fernyhough, C., Jones S. R., Whittle, C., Waterhouse, J., \& Bentall R. P. (2008). Theory of mind, schizotypy, and persecutory ideation in young adults. Cognitive neuropsychiatry, 13(3), 233-49.

Frith, C. D., \& Corcoran, R. (1996). Exploring "theory of mind" in people with schizophrenia. Psychological Medicine, 26(3), 521-530.

Frith, C. D. (1992). The cognitive neuropsychology of schizophrenia. Hove, U.K.: L. Erlbaum.

Gibson, C. M., Penn, D. L., Prinstein, M.J., Perkins, D. O., \& Belger, A. (2010). Social skill and social cognition in adolescents at genetic risk for psychosis. Schizophrenia Research, 122(1-3), 179-184.

Gokcen, S., Bora, E., Erermis, S., Kesikci, H., \& Aydin, C. (2009). Theory of mind and verbal working memory deficits in parents of autistic children. Psychiatry Research, 166(1), 46-53.

Green, M. F., Penn, D. L., Bentall, R., Carpenter, W. T., Gaebel, W., Gur, R. C., ... Heinssen, R.(2008). Social cognition in schizophrenia: An NIMH workshop on definitions, assessment, and research opportunities. Schizophrenia Bulletin, 34(6), 1211-1220.

Happé, F. G. (1994). An advanced test of theory of mind: understanding of story characters' thoughts and feelings by able autistic, mentally handicapped, and normal children and adults. Journal of Autism and Developmental Disorders, 24(2), 129-154.

Janssen, I., Krabbendam, L., Jolles, J., \& van Os, J. (2003). Alterations in theory of mind in patients with schizophrenia and non-psychotic relatives. Acta Psychiatrica Scandinavica, 108(2), 110-117.

Kerr, N., Dunbar, R. I. M., \& Bentall, R. P. (2003). Theory of mind deficits in bipolar affective disorder. Journal of Affective Disorders, 73(3), 253-259.

Lieberman, M. D. (2007). Social cognitive neuroscience: A review of core processes. Annual Review of Psychology, 58, 259-289.

Macrae, C. N., \& Bodenhausen, G. V. (2001). Social cognition:
Categorical person perception. British Journal of Psychology, 92(1), 239-255

Maia, T. V., \& Frank, M. J. (2011). From reinforcement learning models to psychiatric and neurological disorders. Nature Neuroscience, 14(2), 154-162.

Mazza, M., Di Michele, V., Pollice, R., Casacchia, M., \& Roncone, R. (2008). Pragmatic language and theory of mind deficits in people with schizophrenia and their relatives. Psychopathology, 41(4), 254-263.

Meltzoff, A. N. (1999). Origins of theory of mind, cognition and communication. Journal of Communication Disorders, 32(4), 251-269.

Müller, U., \& Liebermann, D. (2004). Facing the body: toward a developmental theory of body knowledge. Monographs of the Society for Research in Child Development, 69(2), 103-113.

Muris, P., Steerneman, P., Meesters, C., Merckelbach, H., Horselenberg, R., van den Hogen, T., \& van Dongen, L. (1999). The TOM Test: A new instrument for assessing theory of mind in normal children and children with pervasive developmental disorders. Journal of Autism and Developmental Disorders, 29(1), 67-80.

Ochsner, K. N., \& Gross, J. J. (2005). The cognitive control of emotion. Trends in Cognitive Sciences, 9(5), 242-249.

Olley, A. L., Malhi, G. S., Bachelor, J., Cahill, C. M., Mitchell, P. B., \& Berk, M. (2005). Executive functioning and theory of mind in euthymic bipolar disorder. Bipolar Disorders, 7(Suppl. 5), 43-52.

Perner, J., Frith, U., Leslie, A. M., \& Leekam, S. R. (1989). Exploration of the autistic child's theory of mind: Knowledge, belief, and communication. Child Development, 60(3), 688-700.

Premack, D., \& Woodruff, G. (1978). Does the chimpanzee have a theory of mind? Behavioral and Brain Sciences, 1(4), 515-526.

Roth, G., \& Dicke, U. (2005). Evolution of the brain and intelligence. Trends in Cognitive Sciences, 9(5), 250-257.

Sabbagh, M. A., Moulson, M. C., \& Harkness, K. L. (2004). Neural correlates of mental state decoding in human adults: An event-related potential study. Journal of Cognitive Neuroscience, 16(3), 415-426.

Sparks, A., McDonald, S., Lino, B., O’Donnell, M., \& Green, M. J. (2010). Social cognition, empathy and functional outcome in schizophrenia. Schizophrenia Research, 122(1-3), 172-178.

Sprong, M., Schothorst, P., Vos, E., Hox, J., \& van Engeland, H. (2007). Theory of mind in schizophrenia: meta-analysis. British Journal of Psychiatry, 191, 5-13.

Striano, T., \& Reid, V. M. (2006). Social cognition in the first year. Trends in Cognitive Sciences, 10(10), 471-476.

Wellman, H. M., \& Liu, D. (2004). Scaling of theory-of-mind tasks. Child Development, 75(2), 523-541.

Wimmer, H., \& Perner, J. (1983). Beliefs about beliefs: Representation and constraining function of wrong beliefs in young children's understanding of deception. Cognition, 13(1), 103-128.

Yatham, L. N., Torres, I. J., Malhi, G. S., Frangou, S., Glahn, D. C., Bearden, C. E., ... Chengappa, K. N. (2010). The International Society for Bipolar Disorders-Battery for Assessment of Neurocognition (ISBD-BANC). Bipolar Disorders, 12(4), 351-363. 